$$
f(x)=\sum_{\mu=1}^{\infty}\left(\log n_{\mu}\right)^{-1 / 4} \phi_{n_{\mu}}(x) .
$$

The details of what is meant by "sufficiently rapidly" are given in $[2$, p. 175]. From what has been shown in $\$ 2$, it follows that for almost every $x$ and for arbitrarily large $n, p_{\nu}$ can be found from our original sequence such that $\left|s_{p_{\nu}}(x ; f)\right| \geqq C_{6}(\log n)^{1 / 4}$. Appropriate modifications can be made in the construction so as to make $f$ real.

\title{
REFERENCES
}

1. G. H. Hardy and W. Rogosinski, Fourier series, Cambridge, 1956.

2. A. Zygmund, Trigonometrical series, Warsaw, 1935.

UNIVERSITY OF CONNECTICUT

\section{A PROBLEM OF RICHARD BELLMAN ${ }^{1}$}

\section{TOMLINSON FORT}

In the January 1957 number of the Bulletin of the American Mathematical Society, Richard Bellman proposes the following problem:

"Consider the Sturm-Liouville problem

$$
\begin{aligned}
u^{\prime \prime}+\lambda[f(x)+\epsilon g(x)] u & =0 \\
u(0)=u(1) & =0
\end{aligned}
$$

where $f(x)$ and $g(x)$ are continuous functions over $[0,1]$ with positive minima.

Let us regard $\lambda_{1}$, the smallest charactistic value as a function of $\epsilon$. Is it true that $\lambda_{1}$, is an analytic function of $\epsilon$ for $R(\epsilon) \geqq 0$ ? In general, where is the singularity nearest the origin $(\epsilon=0)$ ?"

In the present note the method of differences is used.

We shall consider $f(x)$ and $g(x)$ defined as continuous functions $-\eta \leqq x \leqq 1+\eta$ where $\eta>0$ is arbitrarily small. Any definition outside the original interval of definition will do. We shall assume $|f(x)|<N$, $|g(x)|<N$ where $N$ is constant.

Presented to the Society November 29, 1957; received by the editors September $3,1957$.

1 This research was supported by the United States Air Force through the Air Force Office of Scientific Research of the Air Research and Development Command under Contract AF 18(603-23). Reproduction in whole or in part is permitted for any purpose of the United States Government. 
Let $x_{i},=0,1 / n, 2 / n, \cdots, 1$ and consider the difference equation.

$$
n^{2} \Delta^{2} y\left(x_{i}\right)+\lambda\left[f\left(x_{i}\right)+\epsilon g\left(x_{i}\right)\right] y\left(x_{i+1}\right)=0
$$

side by side with (1). We write both (1) and (3) as pairs of equations of the first order. We have

$$
\begin{aligned}
& u_{1}^{\prime}=u_{2}, \\
& u_{2}^{\prime}=-\lambda[f(x)+\epsilon g(x)] u_{1} .
\end{aligned}
$$

We also have

$$
\begin{aligned}
& n \Delta y_{1}\left(x_{i}\right)=y_{2}\left(x_{i}\right), \\
& n \Delta y_{2}\left(x_{i}\right)=-\frac{\lambda}{n}\left[f\left(x_{i}\right)+\epsilon g\left(x_{i}\right)\right] y_{2}\left(x_{i}\right)-\lambda\left[f\left(x_{i}\right)+\epsilon g\left(x_{i}\right)\right] y_{1}\left(x_{i}\right) .
\end{aligned}
$$

We also write (5) in terms of successive values

$$
\begin{aligned}
y_{1}\left(x_{i+1}\right)= & \frac{1}{n} y_{2}\left(x_{i}\right)+y_{1}\left(x_{i}\right), \\
y_{2}\left(x_{i+1}\right)= & -\frac{\lambda}{n^{2}}\left[f\left(x_{i}\right)+\epsilon g\left(x_{i}\right)\right] y_{2}\left(x_{i}\right)+y_{2}\left(x_{i}\right) \\
& -\frac{\lambda}{n}\left[f\left(x_{i}\right)+\epsilon g\left(x_{i}\right)\right] y_{1}\left(x_{i}\right) .
\end{aligned}
$$

If when $n$ is fixed, a solution of (3) is plotted in the cartesian plane, the complete graph consists of isolated points. Connect these points successively with straight line segments, thus forming a "broken" line. The function defined by this continuous graph will be referred to as $y(x)\left[y_{1}(x)\right]$. We know ${ }^{2}$ that $y(x)$ changes sign at each node [zero] and that, if initial conditions are fixed, each node moves continuously to the left as $\lambda$ increases, $\epsilon$ being constant. If $y(0, \lambda, \epsilon)$ is held at zero, the values of $\lambda$ for which $y(1, \lambda, \epsilon)=0$ are characteristic values for equation (3) with boundary conditions $y(0)=y(1)=0$. These values are simple roots of $y(1, \lambda, \epsilon)=0$, which has no other roots.

TheOREM I. Let $u(x, \lambda, \epsilon)$ be a solution of (1) where $u(0, \lambda, \epsilon)=0$ and $\partial u(0, \lambda, \epsilon) / \partial x=1$. Let $y(x, \lambda, \epsilon)$ be a solution of (3) such that $y(0, \lambda, \epsilon)=0$ and $n \Delta_{x} y(0, \lambda, \epsilon)=1$. Then when $n \rightarrow \infty, y(x, \lambda, \epsilon)$ approaches $u(x, \lambda, \epsilon)$ uniformly in $x, \lambda, \epsilon$ so long as $0 \leqq x \leqq 1,|\lambda|<M,|\epsilon|<M$ where $M$ is a constant but otherwise arbitrary. Moreover, $u(x, \lambda, \epsilon)$ and $y(x, \lambda, \epsilon)$ are each expressible as power series in $\lambda$ where, when $n \rightarrow \infty$, the coeff-

2 Tomlinson Fort, Finite differences and difference equations in the real domain, Clarendon Press, 1948. 
cients of the various powers of $\lambda$ in the series for $y$ approach uniformly the coefficients of like powers of $\lambda$ in the power series for $u$.

The proof of this theorem follows so closely well known methods that it is only sketched.

First, we follow the Picard method of successive approximations for $u_{1}$ and $u_{2}$ of system (4). Let successive approximations be $u_{1}^{(0)}, u_{2}^{(0)}$ respectively, $v=0,1, \cdots$. Let $U_{1}^{(0)}(x) \equiv u_{1}^{(0)}(x) \equiv 0, U_{1}^{(v)}(x)=u_{1}^{(0)}(x)$ $-u_{1}^{(v-1)}(x)$ when $v>0$, with similar definitions for $U_{2}^{(v)}(x)$. Then when $v$ is even $U_{1}^{(0)}(x) \equiv 0$. Also $U_{1}^{(1)}(x, \lambda, \epsilon)=x$.

When $v>1$ is odd

$$
U_{1}^{(v)}(x, \lambda, \epsilon)=(-1)^{(v-1) / 2} \int_{0}^{x} \int_{0}^{x_{1}} \lambda\left[f\left(x_{2}\right)+\epsilon g\left(x_{2}\right)\right] \cdots
$$

$$
\begin{array}{r}
\int_{0}^{x_{v-5}} \int_{0}^{x_{v-4}} \lambda\left[f\left(x_{v-3}\right)+\epsilon g\left(x_{v-3}\right)\right] \\
\int_{0}^{x_{v-3}} \int_{0}^{x_{v-2}} \lambda x_{v-1}\left[f\left(x_{v-1}\right)+\epsilon g\left(x_{v-1}\right)\right] d x_{1} d x_{2} \ldots \\
d x_{v-4} d x_{v-3} d x_{v-2} d x_{v-1} .
\end{array}
$$

Hence when $v>1$ is odd.

$$
\left|U_{1}^{(v)}(x, \lambda, \epsilon)\right| \leqq \frac{[\lambda N(1+\epsilon)]^{(v-1) / 2}}{(v-1) !} .
$$

We have

$$
\begin{aligned}
u_{1}(x, \lambda, \epsilon) & \equiv u_{1}(x, \lambda, \epsilon)=x+U_{1}^{(2)}+U_{1}^{(3)}+\cdots \\
& =x+\lambda \widetilde{U}_{3}+\lambda^{2} \widetilde{U}_{5}+\cdots
\end{aligned}
$$

where $\tilde{U}_{3}, \tilde{U}_{5}, \cdots$ are independent of $\lambda$. We now apply the method of successive approximations to $y_{1}(x, \lambda, \epsilon)$. We adopt a notation analogous to that just used. When $v>1$ is odd:

$$
\begin{aligned}
Y_{1}^{(v)}=(-1)^{(v-1) / 2} & \sum_{0}^{x_{i-1}} \sum_{0}^{x(1)_{j-1}} \lambda\left[f\left(x_{i}^{(2)}\right)+\epsilon g\left(x_{i}^{(2)}\right)\right] \cdots \\
& \sum_{0}^{x(v-5)_{i-1}} \sum_{0}^{x(v-4)_{i-1}} \lambda\left[f\left(x_{i}^{(v-3)}\right)+\epsilon g\left(x_{i}^{(v-3)}\right)\right] \\
& \sum_{0}^{x(v-3) i_{i-1}} \sum_{0}^{x(v-2)_{i-1}} \lambda x_{i-1}^{(v-1)}\left[f\left(x_{i}^{(v-1)}\right)+\epsilon g\left(x_{i}^{(v-1)}\right)\right] \frac{1}{n^{v-1}}\left[1+\frac{\phi_{n}}{n}\right]
\end{aligned}
$$

where $\phi_{n}$ is bounded. Here we use the convention $\sum_{i=0}^{v} F(i)=0$, if 
$v<0$. The presence of the term $\phi_{n} / n$ arises from the presence of $\lambda\left[f\left(x_{i}\right)+\epsilon g\left(x_{i}\right)\right] / n$ in (5). We also notice, incidentally, that $Y_{1}^{(o)}\left(x_{i}\right)$ $=0, v>i+1$. We write

$$
y\left(x_{i}, \lambda, \epsilon\right)=x_{i}+\lambda \tilde{Y}_{3}+\lambda^{2} \tilde{Y}_{5}+\cdots
$$

analogously to (9). The conclusions of the theorem are immediate from (9) and (10) since both series converge absolutely and uniformly and $\tilde{Y}_{v}$ approaches $\tilde{U}_{v}$.

We now let $n$ be large and consider the equation

$$
u(1, \lambda, \epsilon)=0 \text {. }
$$

We also let $\bar{\lambda}_{1}$ be the smallest characteristic value for the system consisting of (3) and $y(0, \lambda, \epsilon)=y(1, \lambda, \epsilon)=0$. Now if $y\left(x_{i}, \bar{\lambda}_{1}, \epsilon\right)$ is calculated successively from the initial values $y\left(0, \bar{\lambda}_{1}, \epsilon\right)=0$ and $n \Delta y\left(0, \bar{\lambda}_{1}, \epsilon\right)=1$, we see that $y\left(1, \bar{\lambda}_{1}, \epsilon\right)$ is an integral rational function of $\bar{\lambda}_{1}$ and $\epsilon$ and the term of highest power in $\bar{\lambda}_{1}$ is

$$
\begin{aligned}
\left(\frac{1}{n^{2}}\right)^{n-1}[f(0)+\epsilon g(0)]\left[f\left(x_{1}\right)+\epsilon g\left(x_{1}\right)\right] & \ldots \\
& {\left[f\left(x_{n-2}\right)+\epsilon g\left(x_{n-2}\right)\right]\left(\bar{\lambda}_{1}\right)^{n-1} . }
\end{aligned}
$$

We shall consider the equation

$$
y\left(1, \bar{\lambda}_{1}, \epsilon\right)=0
$$

side by side with (11). Here $\epsilon$ has the same value in both equations. Replace $u(1, \lambda, \epsilon)$ and $y(1, \bar{\lambda}, \epsilon)$ in (11) and (13) by their power series developments already obtained. In particular we notice that $\widetilde{U}_{v}\left(1, \lambda_{1}, \epsilon\right)$ and $\tilde{Y}_{v}\left(1, \bar{\lambda}_{1}, \epsilon\right)$ are each of degree $(v-1) / 2$ in $\epsilon$. We next rearrange (11) and (13) so that terms of like degree considering both $\lambda_{1}$ and $\epsilon$ come together. This is merely rearranging the terms of an absolutely convergent double series. We write

$$
\begin{aligned}
& u(1, \lambda, \epsilon)=A_{0}+A_{1}\left(\lambda_{1}, \epsilon\right)+A_{2}\left(\lambda_{1}, \epsilon\right)+\cdots, \\
& y(1, \bar{\lambda}, \epsilon)=B_{0}+B_{1}\left(\bar{\lambda}_{1}, \epsilon\right)+B_{2}\left(\bar{\lambda}_{1}, \epsilon\right)+\cdots
\end{aligned}
$$

Here $A_{v}\left(1, \lambda_{1}, \epsilon\right)$ and $B_{v}\left(1, \bar{\lambda}_{1}, \epsilon\right)$ are each homogeneous. As a matter of fact, in (14) and (15) we have Taylor's series about the origin for $u\left(1, \lambda_{1}, \epsilon\right)$ and $y\left(1, \bar{\lambda}_{1}, \epsilon\right)$. Both series converge when $|\lambda|<\infty,|\epsilon|<\infty$.

We now solve (14) for $\lambda_{1}$ and (15) for $\bar{\lambda}_{1}$ as functions of $\epsilon$. We denote the functions obtained by $\chi(\epsilon)$ and $\psi_{n}(\epsilon)$. The method employed is the classical majorant method used since the time of Cauchy. The processes are precisely the same for both equations. The same majorant function answers for both and the terms of the series for $\psi_{n}(\epsilon)$ 
approach the corresponding terms in $\chi(\epsilon)$. Hence, according to Abel's continuity theorem $\psi_{n}(\epsilon)$ approaches $\chi(\epsilon)$. Now $\psi_{n}(\epsilon)$ and $\chi(\epsilon)$ are power series. We let these series define the functions for complex values of $\epsilon$ so long as they are convergent. They are then analytically continued. The singularities of $\psi_{n}(\epsilon)$ are the points where the coefficient of the highest power of $\bar{\lambda}_{1}$ in (15) vanishes. This coefficient is given by (12). All of its vanishing points are real and negative. No one is closer to the origin than - minimum $f(x) / g(x)$. Now the singularities of $\chi(\epsilon)$, which is approached uniformly by $\psi_{n}(\epsilon)$ so long as $\epsilon$ is bounded, are either singularities of some $\psi_{n}(\epsilon)$ or a limiting point of such. Hence no one is closer to the origin than

$$
-\min f(x) / g(x) \text {. }
$$

We state the following theorem.

Theorem II. The function $\lambda_{1}=\chi(\epsilon)$ obtained from $u\left(1, \lambda_{1}, \epsilon\right)=0$ for real $\epsilon$ as a power series can be analytically continued over any finite region not including the negative axis of reals. Its singularity nearest the origin is - minimum $f(x) / g(x)$ when $x$ is on $[0,1]$.

University of South Carolina 